\title{
MicroRNA-132-3p regulates cell proliferation, apoptosis, migration and invasion of liver cancer by targeting Sox4
}

\author{
JIANSHENG HUANG ${ }^{*}$, DUDAN LU*, TIANXIN XIANG, XIAOPING WU, \\ SHANFEI GE, YUE WANG, JIAXIN WANG and NA CHENG
}

Department of Infectious Diseases, The First Affiliated Hospital of Nanchang University, Nanchang, Jiangxi 330006, P.R. China

Received May 24, 2019; Accepted December 13, 2019

DOI: $10.3892 / \mathrm{ol} .2020 .11431$

\begin{abstract}
The present study investigated whether microRNA (miR)-132-3p targeted transcription factor SOX-4 (Sox4) for the inhibition of proliferation, migration, invasion and promotion of apoptosis in liver cancer (LC) cells. The expression of miR132-3p and Sox4 mRNA was evaluated by quantitative PCR and protein expression was determined by western blot analysis. Cell proliferation, apoptosis, migration, and invasion were assessed at different time points by the MTT assay, flow cytometry analysis, wound healing assay and Transwell migration assay, respectively. Bioinformatics prediction and luciferase assays were performed to validate and confirm Sox4as a potential target of miR-132p. There was a reduced expression of miR-132-3p in HepG2 and Huh7 cell lines compared with HccLM3 cells. Overexpression of miR-132-3p resulted in significant inhibition of proliferation and induction of apoptosis in LC cells. Moreover, migration and invasion of HepG 2 cells were suppressed by over expressing miR-132-3p. However, downregulation of miR-132-3p in Hep-G2 cells promoted cell growth, invasion and migration and inhibited apoptosis. Bioinformatics analysis predicted Sox4 as a potential target of miR-132-3p, which was further confirmed by the luciferase reporter assay. In addition, an inverse association was observed between miR-132-3p and Sox4 expression. miR-132-3p may regulate the proliferation, apoptosis, migration and invasion of HepG 2 cells by targeting Sox 4 .
\end{abstract}

\section{Introduction}

Liver cancer (LC) is a major cause of morbidity and mortality. Hepatocellular carcinoma ( $\mathrm{HCC})$ is the main histological

Correspondence to: $\mathrm{Dr} \mathrm{Na}$ Cheng, Department of Infectious Diseases, The First Affiliated Hospital of Nanchang University, 17 Yongwai Zheng, Nanchang, Jiangxi 330006, P.R. China

E-mail: chengnah@sina.com

*Contributed equally

Key words: microRNA-132-3p, transcription factor SOX-4, liver cancer, proliferation, apoptosis, migration, invasion subtype of LC, accounting for $\sim 90 \%$ of primary LC, and the third leading cause of cancer-related deaths (1). In 2018, the number of new HCC cases was 841,080 , while the number of deaths was 781,631 (1). The early clinical manifestations of LC are not obvious, and once diagnosed about half are found at an advanced or terminal stage, while LC also has a poor prognosis and the 5-year survival rate is only $16 \%$ worldwide (2). Approximatey three-quarters of LC cases are attributed to chronic hepatitis B virus (HBV) and hepatitis C infections (3). Also, $\sim 50 \%$ of patients with LC have HBV-associated cirrhosis and the morbidity of LC can be as high as $78 \%$ in areas where chronic HBV incidence is high (4). The risk of LC among patients with chronic hepatitis $(\mathrm{CH})$ is a 100 times higher compared with patients without $\mathrm{CH}$ and the incidence of HBV-associated cirrhosis is also higher in patients with $\mathrm{CH}(5,6)$. China has a very high incidence of HBV infection with a high proportion of new cases every year and has become one of the countries with the highest incidence of LC in the world (1). Therefore, strategies that may prevent HBV infection and spread are urgently required. In addition, understanding the mechanisms of proliferation, apoptosis, invasion, and migration of LC cells is important for developing better treatment regimens for patients with $\mathrm{LC}$.

Transcription factor SOX-4 (Sox4) is a $47-\mathrm{kDa}$ protein and a member of the high-mobility group box transcription factor family. The DNA-binding domains of Sox 4 are as follows: i) DNA-binding transcription factor activity, RNA polymerase II-specific; ii) protein heterodimerization activity; and iii) transcription regulatory region sequence-specific DNA binding (7). The Sox4 protein, with a single-box, binds with high sequence specificity to variants of the DNA sequence $(\mathrm{A} / \mathrm{T})(\mathrm{A} / \mathrm{T}) \mathrm{CAAAG}$ resulting in deformation of the DNA molecule to facilitate the binding of other transcription factors capable of binding the afore mentioned DNA sequence (8). Meta-analysis data reported that Sox 4 plays an important role in tumor development (9). Sox 4 has been demonstrated to be highly expressed in human LC samples and to contribute to hepatocarcinogenesis by inhibiting p53-mediated apoptosis, while decreased expression of Sox 4 could be a useful prognostic marker for survival after surgical resection, as low expression levels of Sox 4 could significantly inhibit the growth and migration of LC cells (10). However, the exact mechanism by which Sox 4 regulates $\mathrm{LC}$ development remains unclear. 
MicroRNAs (miRs) are endogenous non-coding RNA molecules of $\sim 22$ nucleotides in length, that function as important regulators of gene expression by binding to the 3' untranslated region (UTR) of specific mRNA molecules (11). Available evidence indicates that dysregulation of miRs can contribute to tumor progression and metastasis $(12,13)$. Human miR-132, one of the miRs that can potentially regulate the expression of various tumor suppressor genes, including $\mathrm{p} 53$, is located on human chromosome $17(14,15)$. The expression of miR-132 in LC, osteosarcoma and colorectal cancer tissue has been demonstrated to be lower compared with normal tissue (14-16). In the present study, bioinformatics analysis predicted Sox 4 as a potential target gene of miR-132 and it was hypothesized that over expression of miR-132 markedly inhibited cancer cell growth, invasion and migration as well as promoted cell apoptosis by targeting Sox 4 .

\section{Materials and methods}

Cell lines and culture. Human LC cell lines HepG2, Huh7 and HccLM3 were obtained from Shanghai Chinese Academy of Sciences. The cells were cultured in Eagle's Minimum Essential Medium (EMEM; Thermo Fisher Scientific Inc.) containing 10\% FBS (Thermo Fisher Scientific, Inc.), 1\% penicillin and streptomycin solution at $37^{\circ} \mathrm{C}$ in an incubator with a humidified atmosphere and $5 \% \mathrm{CO}_{2}$.

Transient transfection for HepG2 cells. miR-132 mimic (5'-ACCGUGGCUUUCGAUUGUUACU-3'), miR-negative control of the mimic (5'-CAGGUAAUCAACGCGGAGGUC A-3'), miR-132 inhibitor (5'-AGUAACAAUCGAAAGCCA CGGU-3') and miR-negative control of the inhibitor (5'-CGU GGUGCUCGUGAAGGGUCGG-3') were synthesized and purified by Suzhou GenePharma Co., Ltd. All miRs above were transfected at a final concentration of $50 \mathrm{nmol} / \mathrm{l}$ using Lipofectamine ${ }^{\circledR} 2000$ reagent (Thermo Fisher Scientific Inc.) following the manufacturer's protocols. The group with untreated cells was defined as control group. Total RNA and protein were extracted $48 \mathrm{~h}$ after transfection.

Reverse transcription-quantitative (RT-q) PCR. Total RNA including miRs was isolated from different LC cell lines using TRIzol $^{\mathbb{B}}$ reagent (Thermo Fisher Scientific, Inc) following the manufacturer's protocol. By using a Prime Script reverse-transcribed Reagent Kit with gDNA Eraser (cat. no. RR047A, Takara Bio, Inc.), total RNA was reverse-transcribed into cDNA. Gene expression levels were determined via real-time PCR using the commercial kit (SYBR Premix Ex Taq ${ }^{\mathrm{TM}}$ II with Tli RNaseH) (cat. no. RR820A, Takara Bio, Inc.,) in an ABI PRISM 7500 system (Thermo Fisher Scientific, Inc.). Thermo cycling conditions were as follows: $95^{\circ} \mathrm{C}$ For $5 \mathrm{~min}$; 40 cycles at $95^{\circ} \mathrm{C}$ for $15 \mathrm{sec} ; 56^{\circ} \mathrm{C}$ for $30 \mathrm{sec}$; and $72^{\circ} \mathrm{C}$ for $15 \mathrm{sec}$ with a final extension at $72^{\circ} \mathrm{C}$ for $7 \mathrm{~min}$. The relative quantification value for each gene was calculated by the $2^{-\Delta \Delta \mathrm{Cq}}$ method (17) using U6 small nuclear RNA as an internal reference gene. The following human-specific primers were used: $\beta$-actin forward, 5'-AGCGAGCATCCCCCAAAG TT-3' and reverse, 5'-GGGCACGAAGGCTCATCATT-3; Sox4, forward, 5'-CAGCAAACCAACAATGCCGA-3' and reverse, 5'-GATCTGCGACCACACCATG-3'; hsa-miR-132-3p forward, 5'-TGCGCTAACAGTCTACAGCCA-3' and loop primer, 5'-GTCGTATCCAGTGCAGGGTCCGAGGTATTC GCACTGGATACGACCGACCATG-3'; U6 forward, 5'-CGC TTCGGCAGCACATATAC-3' and reverse, 5'-AAATATGGA ACGCTTCACGA-3'; miRNA antisense strand, the Universal reverse primer, 5'-CCAGTGCAGGGTCCGAGGTATT-3'. All experiments were performed in triplicate.

Cell proliferation assay. To explore the effect of miR-132-3p on the proliferation of HepG2 cells, $3 \times 10^{3}$ cells were seeded in a 96-well plate and allowed to grow overnight. The cells were then transfected with miRs or control sequences for $48 \mathrm{~h}$. The purple formazan was dissolved using DMSO. MTT cell proliferation assay was performed at 24,48 and $72 \mathrm{~h}$ at a wavelength of $568 \mathrm{~nm}$. Experiments were performed in triplicate.

Flow cytometry for apoptosis analysis. HepG2 cells were transfected with cmiRs for $48 \mathrm{~h}$. Transfected cells were harvested, washed twice in PBS and then stained with Annexin V-allophycocyanin (APC) and 7-aminoactinomycin D (7-AAD) using the Annexin V-APC/7-AAD detection kit (Nanjing KeyGen Biotech Co., Ltd.) following the manufacturer's protocols. The apoptotic cells were positively stained by Annexin V. Each sample was analyzed using a FACSCalibur (BD Biosciences) with Cell Quest Pro software (version 5.1). All experiments were performed in triplicate.

Transwell invasion assay. To determine cell invasion, the Transwell Matrigel invasion assay was conducted using Transwell chambers precoated with Matrigel at $37^{\circ} \mathrm{C}$ for $5 \mathrm{~h}$ (BD Biosciences), following the manufacturer's instructions. Briefly, $2 \times 10^{4} \mathrm{HepG} 2$ cells transfected with miRs were suspended in $150 \mu \mathrm{l}$ of EMEM without serum and seeded on the upper chamber. EMEM (600 $\mu \mathrm{l})$ containing 10\% FBS was added to the lower chamber. After $24 \mathrm{~h}$ incubation at $37^{\circ} \mathrm{C}$ in a $5 \% \mathrm{CO}_{2}$ incubator, cells that remained in the upper chamber were removed with cotton swabs and the penetrating cells were fixed in methanol, and then stained with $0.1 \%$ crystal violet for $20 \mathrm{~min}$ at room temperature. Cell invasion was quantified by counting cells on the lower surface by phase contrast microscopy using an Olympus IX51 microscope (Olympus Corporation). Quantitative analysis of invasion SPSS 17.0 software (SPSS, Inc.)

Cell wound healing assay. Migratory ability of LC cells was determined using the cell wound healing assay. HepG2 cells were plated into six-well plates without antibiotics. Then, cells were transfected with miR-132 mimic, mimic control, miR-132 inhibitor and inhibitor control. After $48 \mathrm{~h}$, transfected cells were digested with $0.25 \%$ pancreatin and $5 \times 10^{5}$ cells were inoculated into six-well plates so that they could form a confluent monolayer by the next day. A $200 \mu$ l pipette head perpendicular to the back of the plates was used to scratch a line, making sure that each well had at least 5 lines. Then, the cells were washed 3 times with PBS and cultured in serum-free medium at $37^{\circ} \mathrm{C}$ in an incubator with $5 \% \mathrm{CO}_{2}$. After $24 \mathrm{~h}$, the cells were visualized and images were captured under an Olympus IX51 phase-contrast inverted microscope (Olympus Corporation). The width of cell wound closure was 
measured at 0 and $24 \mathrm{~h}$ of the experiment. All experiments were performed in triplicate.

Western blot analysis. For protein extraction, HepG2 cells were washed twice in cold PBS and then lysed in RIPA lysis buffer (Beyotime Institute of Biotechnology) containing a protease inhibitor cocktail (Merck KGaA). The protein concentration of cell lysates was quantified using abicinchoninic acid kit (Beyotime Institute of Biotechnology) and $40 \mu \mathrm{g}$ of each of protein extract were separated by $10 \%$ SDS-PAGE, and then transferred to a PVDF membrane. The membranes were blocked with $5 \%$ skimmed milk diluted with Tris-buffered saline Tween-20 at room temperature for $2 \mathrm{~h}$ and incubated overnight at $4^{\circ} \mathrm{C}$ with rabbit anti-Sox 4 antibody (1:500; Boster Biotechnology; cat. no. pb0654) and rabbit anti-GAPDH antibody (1:1,000; cat. no. ab9485; Abcam). The membranes were then incubated with secondary antibody (1:1,000; cat. no. A0277; Beyotime Institute of Biotechnology) for $2 \mathrm{~h}$ at room temperature. The protein bands were visualized using ECL plus reagents (Thermo Fisher Scientific, Inc.). The densitometry of the western blot protein bands was measured using the BandScan version 5.0 software (Glyko Biomedical, Ltd). Each sample was analyzed in triplicate.

Vector construction. The Psi-Check-Sox4 full length 3'UTR plasmid (Addgene Plasmid \#26989) was digested with the NotI and XhoI enzymes to obtain the full-length 3'UTR of Sox4. The recombinant vector was termed as pYr-MirTarget (Biovector Lab, Inc.). All the constructs were verified by sequencing.

Dual-luciferase reporter assay. HepG2 cells ( $2 \times 10^{5} /$ well) were seeded in 12-well plates. The next day cells were co-transfected with $1 \mu \mathrm{g}$ pYr-MirTarget-Sox4-3'UTR reporter plasmid, $50 \mathrm{nmol} / 1$ of miR132-3p mimic or mimic control using Lipofectamine 2000. Then, $24 \mathrm{~h}$ after transfection, both Firefly and Renilla luciferase activities were quantified using the dual luciferase reporter system (Beyotime Institute of Biotechnology) according to the manufacturer's instructions. The relative luciferase unit (Renilla luciferase/Firefly luciferase) was calculated to determine the activation of the target gene. All experiments were performed at least twice.

Bioinformatic analysis. To determinewhether Sox 4 is a direct target of miR-132-3p, two bioinformatic software programs were used, TargetScan.human6.2 (targetscan.org/vert_61/) and microRNA.org August 2010 release (microrna. org/microrna/home.do).

Statistical analysis. Statistical analysis was performed using the SPSS 17.0 software (SPSS, Inc.). The data were presented as the mean \pm standard deviation. One-way analysis of variance followed by Tukey's or Bonferroni's post hoc test was used for multiple comparisons between the groups. $\mathrm{P}<0.05$ was considered to indicate a statistically significant difference.

\section{Results}

Expression of miR-132-3p is downregulated in human LC cell lines. First, the basal expression of miR-132-3p was determined by RT-qPCR in three liver cancer cell lines (HepG2,

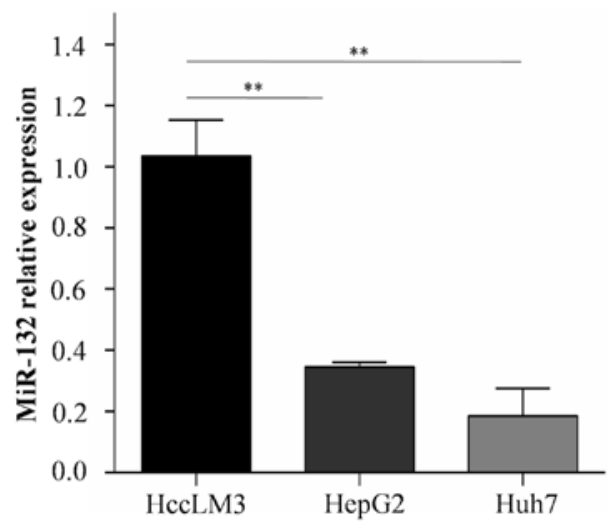

Figure 1. mRNA expression of miR-132-3p inHepG2, Huh7 and HccLM3 liver cancer cell lines. ${ }^{* *} \mathrm{P}<0.01$. miR, microRNA.

Huh7 and HccLM3). The results demonstrated significantly reduced expression of miR-132-3p in HepG2 and Huh7cell lines compared with the HccLM3 cell line (Fig. 1). Among these 3 cell lines, HepG2 was chosen for further experiments due to its common usage.

An inverse association between the expression of $m i R-132-3 p$ and Sox4 in HepG2 cells. As presented in Fig. 2, the level of miR-132-3p was significantly increased following transfection with miR-132-3p mimics compared with the mimics control group confirming the successful over expression of miR-132-3p in HepG2 cells. By contrast, transfection of HepG2 cells with the miR-132-3p inhibitor decreased the expression level of miR-132-3p (Fig. 2A). In addition, the mRNA levels of Sox4 were measured in HepG2 cells as describe above. Sox 4 mRNA expression was decreased in cells overexpressing miR-132-3p, whereas Sox 4 mRNA expression levels were increased in cells under expressing miR-132-3p, compared with the respective control groups (Fig. 2B). Protein expression levels of Sox4 were measured in the control, mimic and inhibitor groups by western blotting. Similar results to those for Sox 4 mRNA expression levels were obtained (Fig. 2C and D).

miR-132-3p inhibitscell proliferation and inducesapoptosis in HepG2 cells. After confirming the expression of miR-132-3p in HepG2 cells, the role of miR-132-3p in proliferation and apoptosis of HepG2 cells was investigated. Cell proliferation measured using the MTT assay revealedthat overexpression of miR-132-3p significantly inhibited the proliferation of HepG2 cells, whereas decreased expression of miR-132-3p promoted the proliferation of HepG2 cells at time points 48 and $72 \mathrm{~h}$ (Fig. 3A). To determine if proliferation was inhibited due to cell apoptosis, apoptosis in HepG2 cells transfected with miR-132-3p mimics or miR-132-3p inhibitor was also measured. Flow cytometry analysis revealed that the number of apoptotic HepG2 cells was significantly higher in cells transfected with miR-132-3p and was significantly lower in cells transfected with the miR-132-3p inhibitor, compared with their respective controls (Fig. 3B and C), thus confirming that miR-132-3p regulated the proliferation and apoptosis of HepG2 cells.

miR-132-3p inhibits invasion and migration of HepG2 cells. After transfection for $48 \mathrm{~h}$, the effects of miR-132 on 

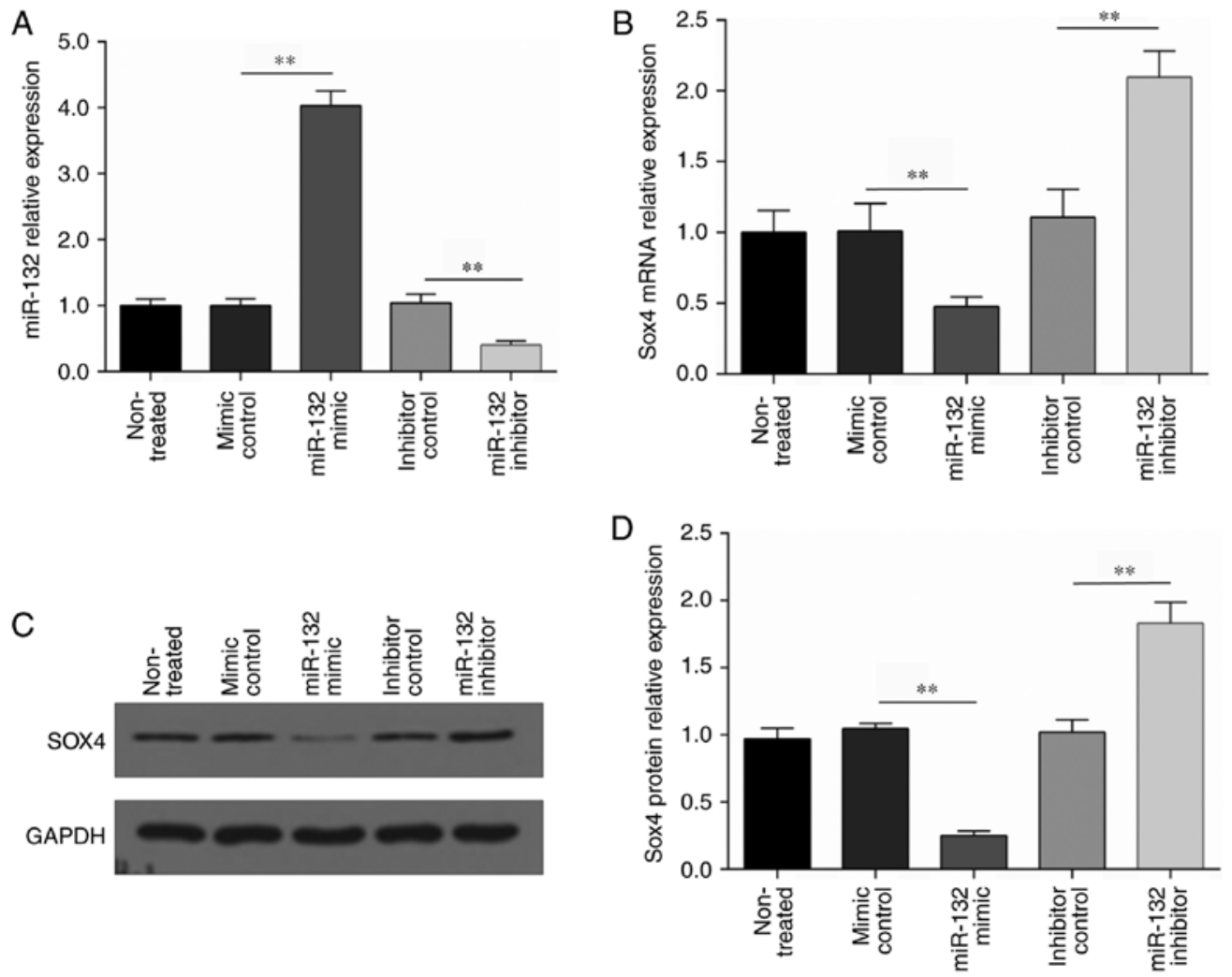

Figure 2. Expression of miR-132-3p and Sox4 in HepG2 cells in five experimental groups. (A) miR-132-3p mRNA expression. (B) Sox4 mRNA expression. (C) Sox4 protein analysis using western blotting and (D) quantification of Sox 4 relative protein expression. ${ }^{* *} \mathrm{P}<0.01$. miR, microRNA; Sox4, transcription factor SOX-4.

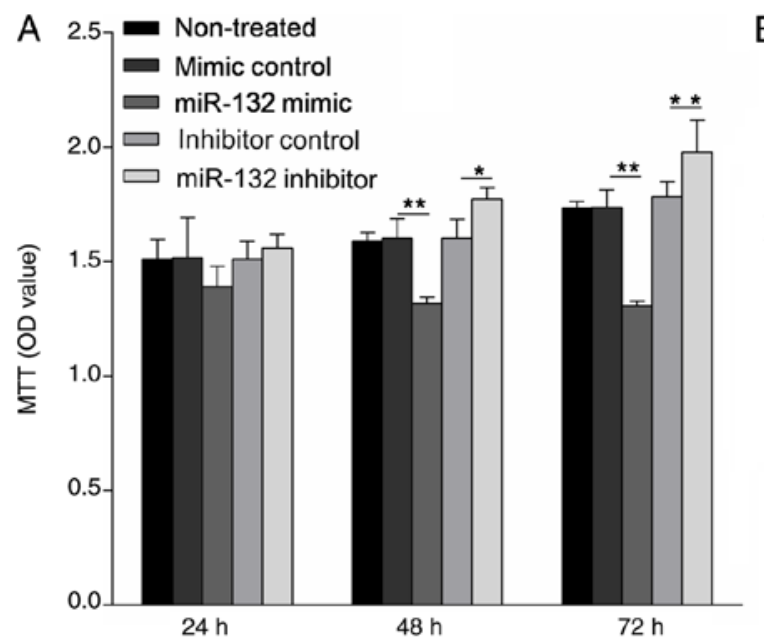

B
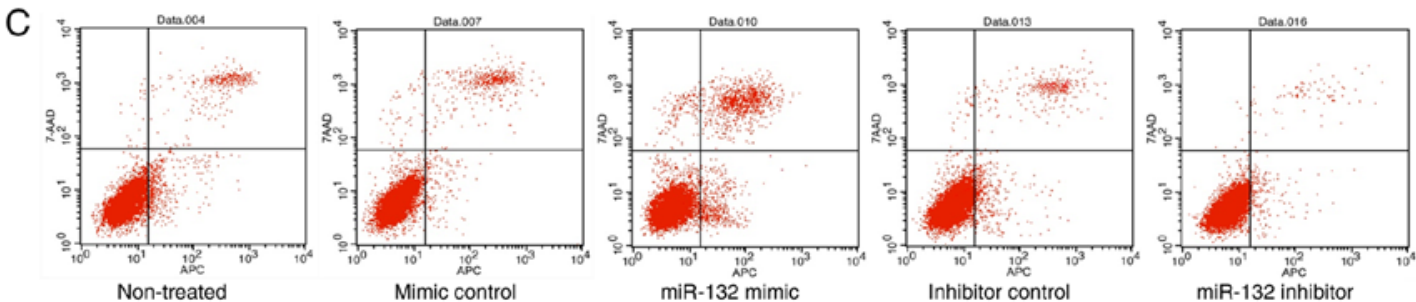

Figure 3. Effect of miR-132-3p expression on (A) proliferation and (B) apoptosis. (C) Representative flow cytometry plots for investigating apoptosis of HepG2 cells using Annexin V-7AAD staining. ${ }^{*} \mathrm{P}<0.05$ and ${ }^{* *} \mathrm{P}<0.01$. APC, allophycocyanin; 7-AAD, 7 -aminoactinomycin D; miR, microRNA.

invasion in HepG2 cells were evaluated using the Transwell invasion chamber assay. The number of invading HepG2 cells was significantly reduced in cells transfected with miR-132-3p mimics compared with those transfected with 
A

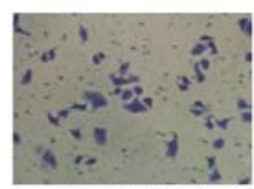

Non-treated

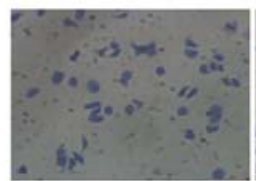

Inhibitor control

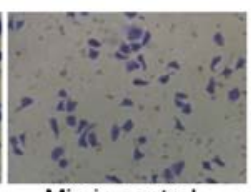

Mimic control

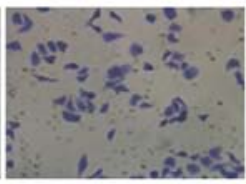

miR-132 inhibitor

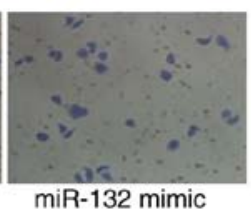

miR-132 mimic
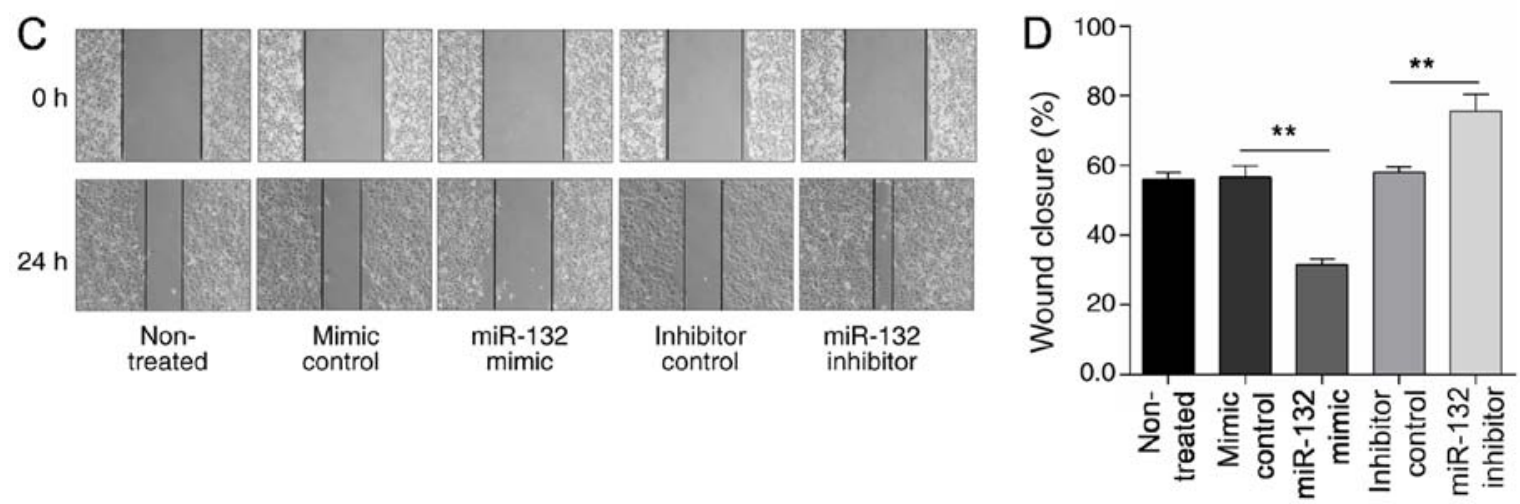

Figure 4. Effects of miR-132-3p on invasion and migration of HepG2 cells. (A) Representative images of invading cells of each groupand (B) quantification of invading cells. (C) Representative images of cell wound healing assay of each experimental group at 0 and 24 h. (D) Percent wound closure of each experimental group. ${ }^{* *} \mathrm{P}<0.01$. miR, microRNA.

mimic control (Fig. 4A and B). By contrast, the number of invading cells was significantly increased in HepG2cells transfected with the miR-132-3p inhibitor, indicating that miR-132-3p inhibited invasion in HepG2 cells (Fig. 4A and B). The wound healing assay results demonstrated that the percent wound closure in the miR-132-3p mimics group was significantly reduced, whereas it was significantly increased in the miR-132-3p inhibitor group compared with the inhibitor control group (Fig. 4C and D). Taken together, the results indicated that miR-132-3p inhibited the migration of HepG2 cells.

Sox4 is a direct target of miR-132-3p in LC cells. The bioinformatic analysis indicated that the Sox43'UTR harbors one conserved binding site (TargetScan.human) and two targets of miR-132-3p (microRNA.org) (Fig. 5A). These sites were at least partially complementary to a motif that is found in the seed region of the miR-132-3p. To confirm thatSox4isa direct target of miR-132-3p, the 3'UTR was cloned to the downstream of the wild type luciferase stop codon. The results revealed that overexpression of miR-132-3p significantly inhibited the luciferase activity of the pUC57-Sox4-3'UTR (Fig. 5B). Together, the results of the bioinformatics analysis and luciferase reporter assay suggested that Sox 4 was a direct target of miR-132-3p in LC cells.

\section{Discussion}

Emerging evidence suggests that miR expression is dysregulated in human malignancies including LC (18). Dysregulated
miRs affect the hallmarks of cancer through the regulation of tumor cell proliferation, apoptosis and other important pathological processes by targeting multiple genes and signaling pathways in LC (18). Among the miRs, miR-132-3pexpression was demonstrated to be more frequently downregulated in HBV-associated LC tissues (19). Additionally, miR-132 was found to be downregulated in LC compared with paracarcinoma and normal liver tissue $(19,20)$. In the present study it was further confirmed that miR-132-3p expression was significantly reduced in HepG2 compared with HccLM3 cells. The mechanism underlying this decreased expression is believed to be mediated through the HBV X protein (HBx)-induced hypermethylation of the promoter of miR-132, which was previously demonstrated to be more prevalent in HBx-expressing HepG2 cells (21). Low expression of miR-132 has been observed in other types of human cancer, including colorectal, lung, cervical, breast cancers and glioma (22-25). By contrast, high miR-132 expression has been reported in gastric cancer (26). The mean level of miR-132 in LC tissues was significantly lower than that found in matched tumor-adjacent tissues and its expression was negatively associated with tumor differentiation and the TNM stage, which is a cancer staging notation system that describes the stage of a cancer which originates from a solid tumor with alphanumeric codes and lymph node metastasis (21). Additionally in the aforementioned study, miR-132 inhibited tumor growth, volume and weight (20). However the precise role and mechanism through which miR-132 exerts its effect on LC was unclear. In the present study, the biological 
A

\author{
TargetScanhuman.6.2 \\ Position 1148-1154 of SOX4 3' UTR $\quad$ 5' ...AAGAUUUCUGUAUAAGACUGUUG... \\ Has-miR-132 \\ |||||| \\ 3'... GCUgGuacCGACAUCUGACAAU...
}

MicroRNA.org

Has-miR-132 3' GCUgGuaccGacAuCugACAAU 5'

SOX4 939: ' CGuggaggagaggagacuguUU 3'

B

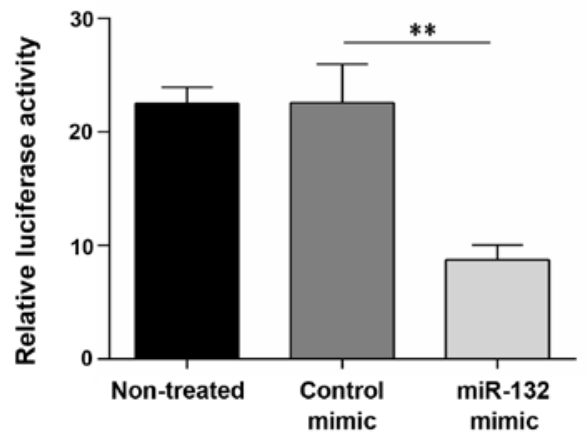

Figure 5. Sox4 is a direct target of miR-132-3p in HepG2 cells. (A) Result of bioinformatics prediction in TargetScan.human 6.2. Conserved binding site found in Sox4 3'UTR locus by TargetScan.human or by microRNA.org. (B) Relative luciferase unit (Renilla luciferase/Firefly luciferase) to detect the activation of Sox4 gene. ${ }^{* *} \mathrm{P}<0.01$. Sox 4 , transcription factor; SOX-4. miR, microRNA; 3'UTR, 3' untranslated region.

functions and mechanism by which miR-132-3p regulates LC were elucidated. Furthermore, to the best of the authors' knowledge, the current study was the first to demonstrate that Sox4 was a novel target of miR-132-3p in LC.

In the present study, miR-132-3p mimics and anmiR-132-3p inhibitor were used to modulate the expression of miR-132-3p in HepG2 cells. The results of the MTT assays demonstrated that overexpression of miR-132-3p inhibited the proliferation of HepG2 cells. Consistent with the results of the current study, Liu et al (20) reported that ectopic expression of miR-132 inhibited cell proliferation, colony formation, migration and invasion, as well as induced apoptosis in HepG2 cells in vitro. Liu et al also demonstrated that miR-132 inhibited LC growth and decreased cancer volume and weight in vivo. In addition, proliferation and colony formation of LC cells were revealed to be suppressed by the miR-132-mediated inhibition of the Akt-signaling pathway in miR-132-transfected cells (26). The present study demonstrated that high levels of miR-132 significantly induced apoptosis of HepG2 cells. However, cell proliferation was promoted and cell apoptosis was inhibited in HepG2 cells transfected with the miR-132-3p inhibitor compared with the inhibitor control group. Moreover, invasion and migration of $\mathrm{HepG} 2$ cells were inhibited by the overexpression of miR-132-3p, which was reversed by miR-132-3p inhibitor transfection. The molecular mechanism underlying this beneficial effect of miR-132-3p was investigated by undertaking the identification and validation of a potential target of miR-132-3p.

Sox 4 expression was reported to be increased in $63.8 \%$ of human HCC tissue samples and the increased expression of Sox4 promoted HCC development (10). Since Sox4 has been demonstrated to be overexpressed in HCC and to contribute to hepatocarcinogenesis, the present study sought to determine whether Sox 4 was a direct target of miR-132-3p in LC cells. This was first determined through bioinformatic analysis using two independent bioinformatic software tools. Furthermore, the present study determined that the expression of Sox 4 mRNA and protein in HepG2 cells was inversely proportional to the level of miR-132-3p. These findings are consistent with previous findings in osteosarcoma and lung cancer cells indicating that Sox4 is a target of miR-132-3p $(27,28)$. Moreover, it has been reported that miR-132-3p inhibited cell growth and metastasis in osteosarcoma cells by downregulating Sox 4 , and knockdown ofSox 4 promoted miR-132-mediated cell growth and metastasis in osteosarcoma cells (27). Similar to the results of the present study, the expression of miR-132 in non-small cell lung carcinoma cells was decreased, and overexpression of miR-132 inhibited cell invasion and migration by targeting SOX4 (28).

Zheng et al (29) reported that overexpression of miR-132 in colorectal cancer cells inhibited cell invasion and migration by targeting the zinc finger E-box binding homeobox 2 gene. Another report indicated that miR-132 regulated apoptosis of glioma cells by blocking the sterol regulatory element-binding transcription factor 1 metabolic pathway related to Sirtuin 1 (30). Studies have also reported that overexpression of Sox 4 was closely associated with tumor progression and metastasis $(31,32)$. Sox 4 maybe a crucial oncogene affecting tumor progression and metastasis in HCC and lung cancer $(10,33)$. Overexpression of Sox 4 has also been detected in other types of solid tumors including prostate, breast, bladder and lung cancer $(7,34,35)$. Downregulation of the expression of Sox 4 inhibited cell proliferation, metastasis and induced apoptosis in lung cancer cells (33).

Since one miR can target multiple genes, it was important to determine whether miR-132-3p targeted other genes besides Sox 4 in the same and in different signaling pathways. In this respect, proliferation and colony formation of LC cells were suppressed through the miR-132-mediated inhibition of the AKT-signaling pathway in miR-132-transfected cells (26). Furthermore, miR-132 
expression was inversely associated with PIK3R3 mRNA expression levels in clinical HCC tissue samples (20). Yes-associated protein 1 is also a potential target of miR-132 (19). The involvement of these targets in a similar signaling pathway in miR-132-3p-mediated regulation of LC warrants further investigation.

Taking the findings of the present study together, it can be hypothesized that miR-132-3p maybe a potential antioncogene in liver cancer. In addition, the present study demonstrates that miR-132-3p serves an important role in regulating proliferation, apoptosis, invasion and migration in LC cells. Further studies are required to evaluate the effects of miR-132-3p in animal models of LC as well as in human LC tissue specimens, blood and plasma samples. The present study provides new insights into the molecular mechanisms mediating the development of human LC. The regulation of miR-132-3p-targeted genes in patients with LC may be an efficacious therapeutic strategy. Additionally, it may enable the screening of early stage LC by determining the level of miR-132-3p in human blood.

\section{Acknowledgements}

Not applicable.

\section{Funding}

This study was supported by grants from the National Natural Science Foundation of China (grant. no. 81760115), Chinese Foundation for Hepatitis prevention and Control-TianQing Liver Disease Research Fund (grant. no. TQGB20170052).

\section{Availability of data and materials}

All data generated or analyzed during this study are included in this published article.

\section{Authors' contributions}

JH, DL, TX and SG performed the experiments. XW and YW analyzed the data. JH, JW and NC contributions to conception and design, and drafting the manuscript or revising it critically for important intellectual content. All authors read and approved the final manuscript.

\section{Ethics approval and consent to participate}

Not applicable.

\section{Patient consent for publication}

Not applicable.

\section{Competing interests}

The authors declare that they have no competing interests.

\section{References}

1. Bray F, Ferlay J, Soerjomataram I, Siegel RL, Torre LA and Jemal A: Global cancer statistics 2018: GLOBOCAN estimates of incidence and mortality worldwide for 36 cancers in 185 countries. CA Cancer J Clin 68: 394-424, 2018.
2. Siegel R, Naishadham D and Jemal A: Cancer statistics, 2013. CA Cancer J Clin 63: 11-30, 2013.

3. Yang JD and Roberts LR: Hepatocellular carcinoma: A global view. Nat Rev Gastroenterol Hepatol 7: 448-458, 2010.

4. Forner A, Llovet JM and Bruix J: Hepatocellular carcinoma Lancet 379: 1245-1255, 2012.

5. Sherman M: Hepatocellular carcinoma: Epidemiology, surveillance, and diagnosis. Semin Liver Dis 30: 3-16, 2010.

6. Beasley RP, Hwang LY, Lin CC and Chien CS: Hepatocellular carcinoma and hepatitis B virus. A prospective study of 22707 men in Taiwan. Lancet 2: 1129-1133, 1981.

7. Grimm D, Bauer J, Wise P, Krüger M, Simonsen U, Wehland M, Infanger M and Corydon TJ: The role of SOX family members in solid tumours and metastasis. Semin Cancer Biol: Mar 23, 2019 (Epub ahead of print). doi: 10.1016/j.semcancer.2019.03.004.

8. van Houte LP, Chuprina VP, van der Wetering M, Boelens R, Kaptein $\mathrm{R}$ and Clevers $\mathrm{H}$ : Solution structure of the sequence-specific HMG box of the lymphocyte transcriptional activator Sox-4. J Biol Chem 270: 30516-30524, 1995.

9. Rhodes DR, Yu J, Shanker K, Deshpande N, Varambally R, Ghosh D, Barrette T, Pandey A and Chinnaiyan AM: Large-scale meta-analysis of cancer microarray data identifies common transcriptional profiles of neoplastic transformation and progression. Proc Natl Acad Sci USA 101: 9309-9314, 2004.

10. Hur W, Rhim H, Jung CK, Kim JD, Bae SH, Jang JW, Yang JM, Oh ST, Kim DG, Wang HJ, et al: SOX4 overexpression regulates the p53-mediated apoptosis in hepatocellular carcinoma: Clinical implication and functional analysis in vitro. Carcinogenesis 31: 1298-1307, 2010.

11. Treiber T, Treiber N and Meister G: Regulation of microRNA biogenesis and function. Thromb Haemost 107: 605-610, 2012.

12. Jafri MA, Al-Qahtani MH and Shay JW: Role of miRNAs in human cancer metastasis: Implications for therapeutic intervention. Semin Cancer Biol 44: 117-131, 2017.

13. Kogure A, Kosaka N and Ochiya T: Cross-talk between cancer cells and their neighbors via miRNA in extracellular vesicles: An emerging player in cancer metastasis. J Biomed Sci 26: 7, 2019.

14. Zhao DW, Hou YS, Sun FB, Han B and Li SJ: Effects of miR-132 on proliferation and apoptosis of pancreatic cancer cells via Hedgehog signaling pathway. Eur Rev Med Pharmacol Sci 23: 1978-1985, 2019

15. Chen Y, Zhu H, Wang Y, Song Y, Zhang P, Wang Z, Gao J, Li Z and Du Y: MicroRNA-132 plays an independent prognostic role in pancreatic ductal adenocarcinoma and acts as a tumor suppressor. Technol Cancer Res Treat 18: 1533033818824314 , 2019.

16. Liu Y and Zhang M: MiR-132 regulates adriamycin resistance in colorectal cancer cells through targeting extracellular signal-regulated kinase 1. Cancer Biother Radiopharm 4: 398-404, 2019.

17. Livak KJ and Schmittgen TD: Analysis of relative gene expression data using real-time quantitative PCR and the 2(-Delta Delta C(T)) method. Methods 25: 402-408, 2001.

18. Song K, Han C, Zhang J, Lu D, Dash S, Feitelson M, Lim K and $\mathrm{Wu}$ T: Epigenetic regulation of MicroRNA-122 by peroxisome proliferator activated receptor-gamma and hepatitis $b$ virus $\mathrm{X}$ protein in hepatocellular carcinoma cells. Hepatology 58: 1681-1692, 2013.

19. Lei CJ, Li L, Gao X, Zhang J, Pan QY, Long HC, Chen CZ, Ren DF and Zheng G: Hsa-miR-132 inhibits proliferation of hepatic carcinoma cells by targeting YAP. Cell Biochem Funct 33: 326-333, 2015.

20. Liu K, Li X, Cao Y, Ge Y, Wang J and Shi B: MiR-132 inhibits cell proliferation, invasion and migration of hepatocellular carcinoma by targeting PIK3R3. Int J Oncol 47: 1585-1593, 2015.

21. Wei X, Tan C, Tang C, Ren G, Xiang T, Qiu Z, Liu R and Wu Z: Epigenetic repression of miR-132 expression by the hepatitis $B$ virus $x$ protein in hepatitis B virus-related hepatocellular carcinoma. Cell Signal 25: 1037-1043, 2013

22. Kara M, Yumrutas O, Ozcan O, Celik OI, Bozgeyik E, Bozgeyik I and Tasdemir S: Differential expressions of cancer-associated genes and their regulatory miRNAs in colorectal carcinoma. Gene 567: 81-86, 2015.

23. Wang H, Li XT, Wu C, Wu ZW, Li YY, Yang TQ, Chen GL, Xie XS, Huang YL, Du ZW and Zhou YX: MiR-132 can inhibit glioma cells invasion and migration by target MMP16 in vitro. Onco Targets Ther 8: 3211-3218, 2015.

24. Zhang H, Liu A, Feng X, Tian L, Bo W, Wang $\mathrm{H}$ and $\mathrm{Hu} \mathrm{Y}$ : MiR-132 promotes the proliferation, invasion and migration of human pancreatic carcinoma by inhibition of the tumor suppressor gene PTEN. Prog Biophys Mol Biol 148: 65-72, 2017. 
25. Zhou K, Zhang C, Yao H, Zhang X, Zhou Y, Che Y and Huang Y: Knockdown of long non-coding RNA NEAT1 inhibits glioma cell migration and invasion via modulation of SOX2 targeted by miR-132. Mol Cancer 17: 105, 2018.

26. Wu WK, Lee CW, Cho CH, Fan D, Wu K, Yu J and Sung JJ: MicroRNA dysregulation in gastric cancer: A new player enters the game. Oncogene 29: 5761-5771, 2010.

27. Liu Y, Li Y, Liu J, Wu Y and Zhu Q: MicroRNA-132 inhibits cell growth and metastasis in osteosarcoma cell lines possibly by targeting Sox4. Int J Oncol 47: 1672-1684, 2015.

28. Li Y, Zu L, Wang Y, Wang M, Chen P and Zhou Q: MiR-132 inhibits lung cancer cell migration and invasion by targeting SOX4. J Thorac Dis 7: 1563-1569, 2015.

29. Zheng YB, Luo HP, Shi Q, Hao ZN, Ding Y, Wang QS, Li SB, Xiao GC and Tong SL: miR-132 inhibits colorectal cancer invasion and metastasis via directly targeting ZEB2. World J Gastroenterol 20: 6515-6522, 2014.

30. Li Y, Zhang J, He J, Zhou W, Xiang G and Xu R: MicroRNA-132 cause apoptosis of glioma cells through blockade of the SREBP-1c metabolic pathway related to SIRT1. Biomed Pharmacother 78 177-184, 2016.

31. Wang L, Zhang J, Yang X, Chang YW, Qi M, Zhou Z, Zhang J and Han B: SOX4 is associated with poor prognosis in prostate cancer and promotes epithelial-mesenchymal transition in vitro. Prostate Cancer Prostatic Dis 16: 301-307, 2013.
32. Zhang H, Alberich-Jorda M, Amabile G, Yang H, Staber PB, Di Ruscio A, Welner RS, Ebralidze A, Zhang J, Levantini E, et al: Sox 4 is a key oncogenic target in $\mathrm{C} / \mathrm{EBP} \alpha$ mutant acute myeloid leukemia. Cancer Cell 24: 575-588, 2013.

33. Zhou Y, Wang X, Huang Y, Chen Y, Zhao G, Yao Q, Jin C, Huang Y, Liu X and Li G: Down-regulated SOX4 expression suppresses cell proliferation, metastasis and induces apoptosis in Xuanwei female lung cancer patients. J Cell Biochem 116: 1007-1018, 2015.

34. Zhang J, Liang Q, Lei Y, Yao M, Li L, Gao X, Feng J, Zhang Y, Gao H, Liu DX, et al: SOX4 induces epithelial-mesenchymal transition and contributes to breast cancer progression. Cancer Res 72: 4597-4608, 2012.

35. Castillo SD, Matheu A, Mariani N, Carretero J, Lopez-Rios F, Lovell-Badge R and Sanchez-Cespedes M: Novel transcriptional targets of the SRY-HMG box transcription factor SOX4 link its expression to the development of small cell lung cancer. Cancer Res 72: 176-186, 2012.

(c) (9) This work is licensed under a Creative Commons Attribution 4.0 International (CC BY-NC 4.0) License 